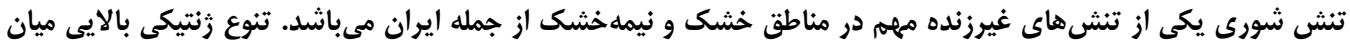

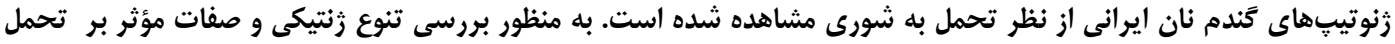

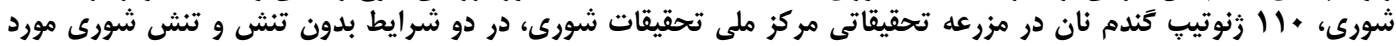

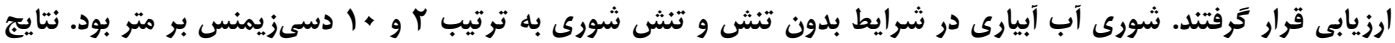

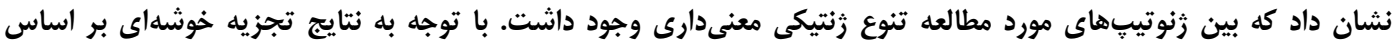

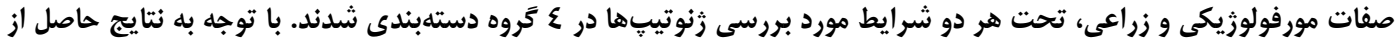

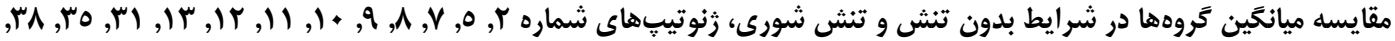

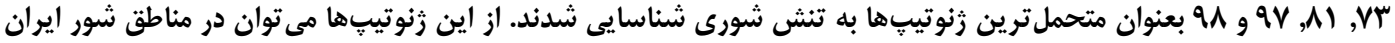

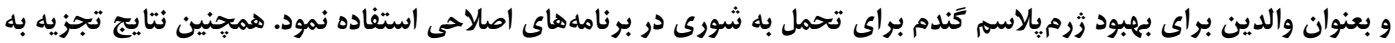

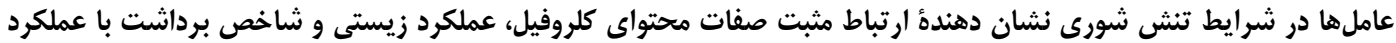

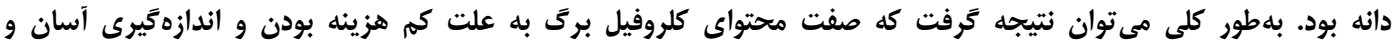

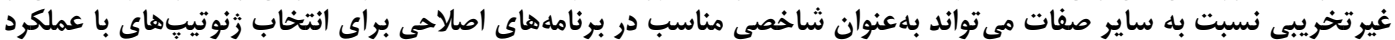
بالا در شرايط تنش شورى در مزرعه مورد استفاده قرار تيردات

وازههاى كليدى: كندم، تنوع زنتيكى، تنش شورى، تجزيه عاملها، تجزيه خوشهاى

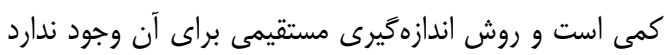

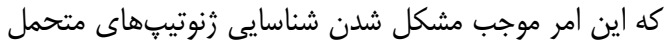

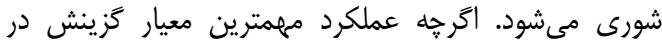

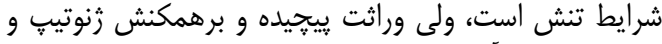

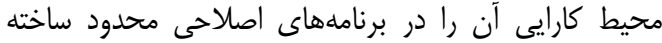

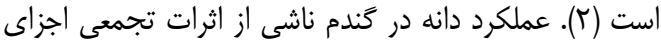

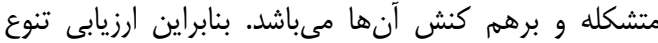

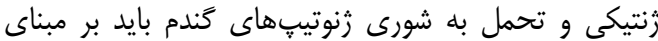

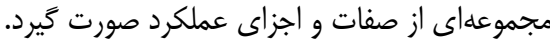

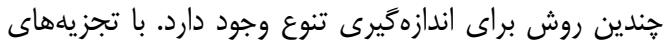

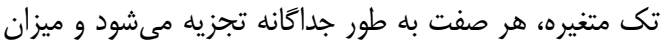

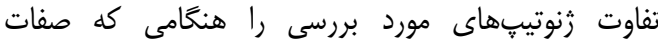

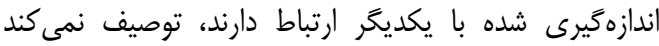

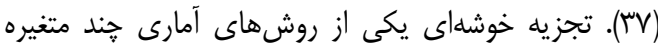

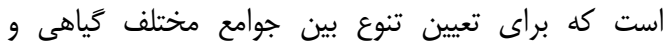

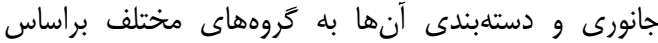

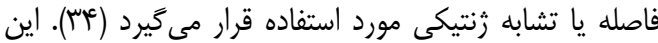

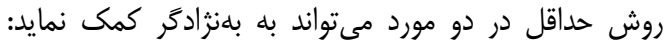

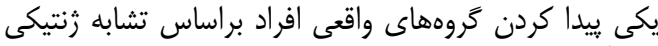

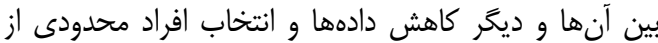

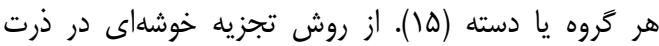

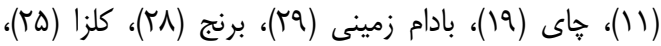

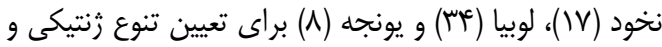

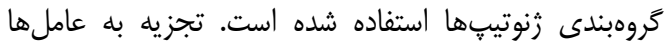

كندم بلهعنوان يك محصول استراتزيك سهم عمدهاى از از

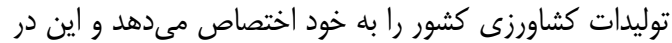

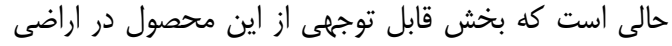

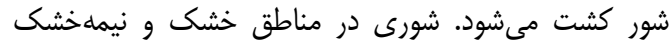

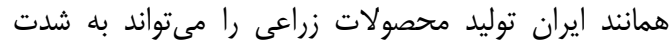

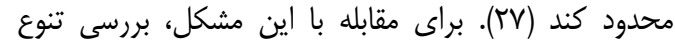

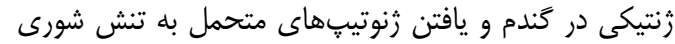

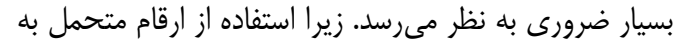

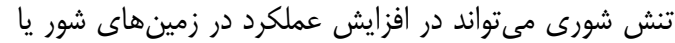

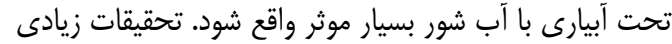

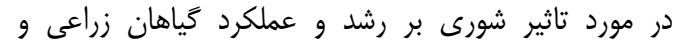

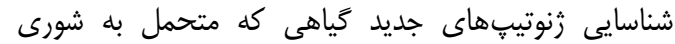

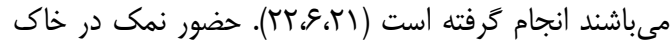

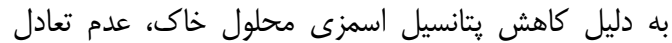

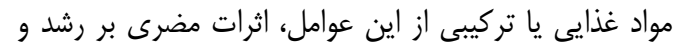

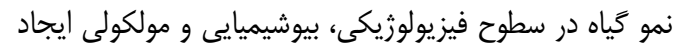

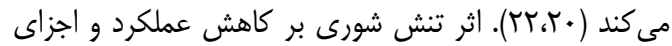

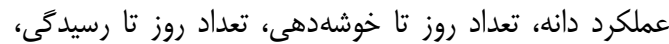

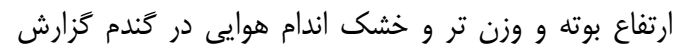

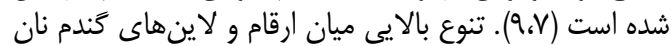

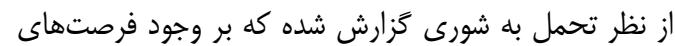

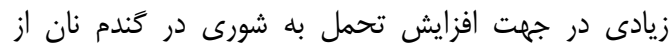

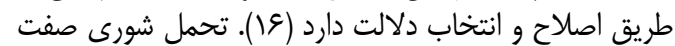




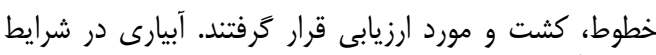

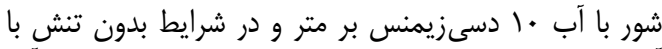

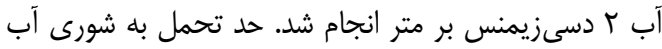

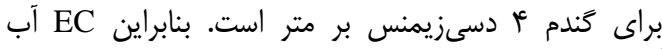

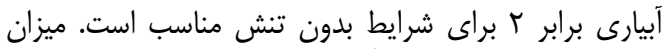

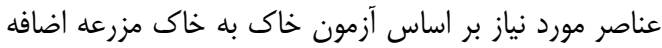

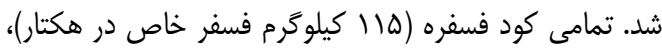

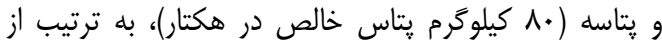

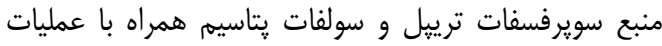

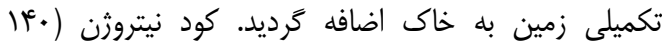

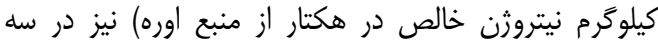

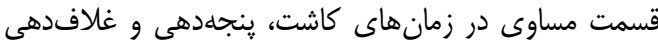

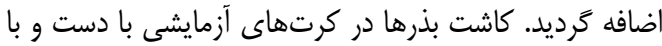

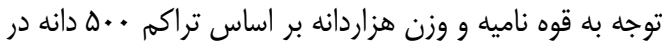

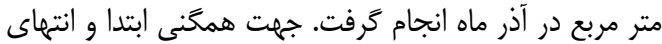

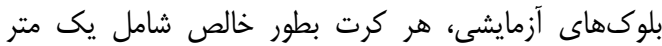

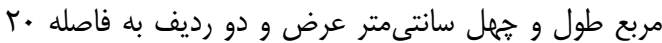

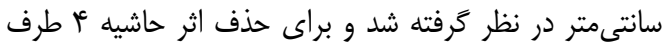

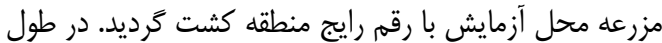

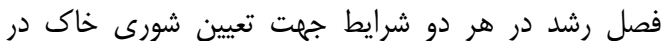

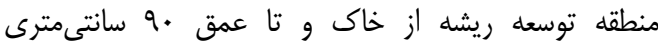

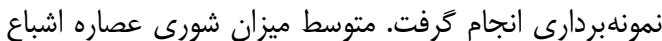

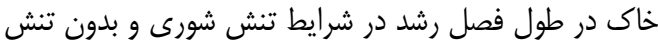

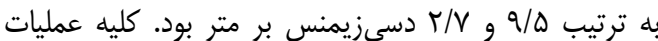

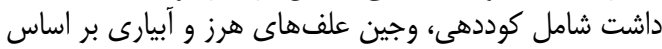

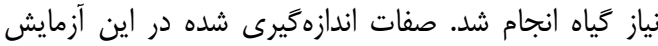

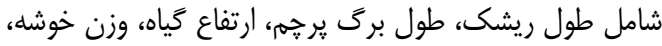

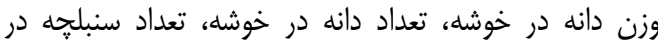

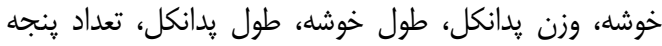

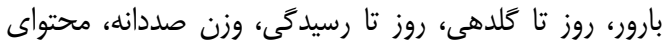

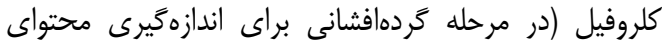

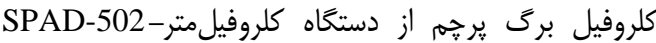

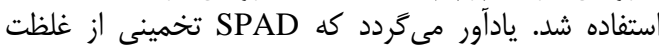

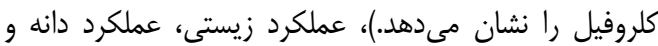
شاخص برداشت بودند. تجزيه و تحليل آمارى نودئ

ابتدا نرمال بودن دادهها از طارئ طريق آزمون كولموركروف

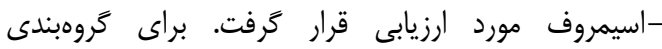

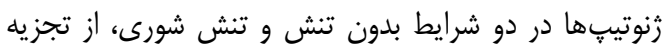

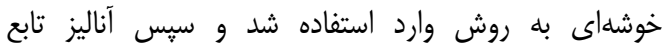

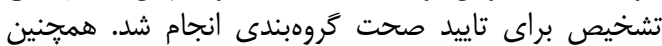

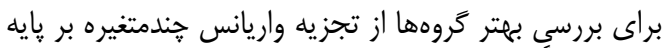

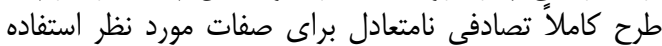

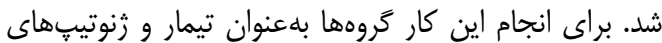

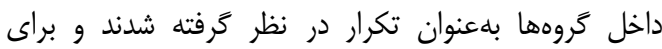

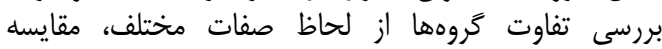

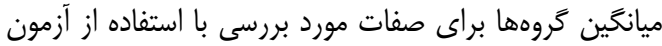

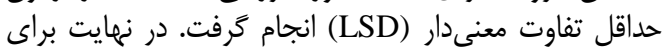

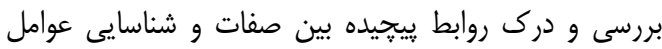

يكى ديخر از روشهاى آمارى جند متغيره است كه بلمنظظور

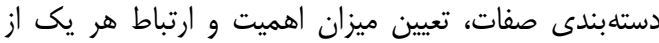

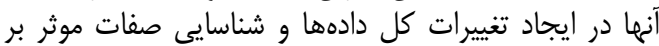

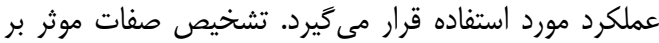

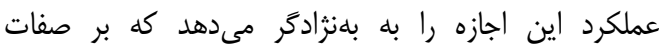

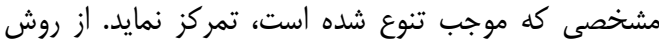

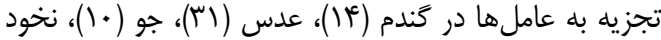

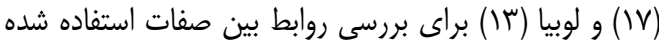

ارزيابى تنوع زنتيكى و بررسى روابط بين صفات بر مبناى

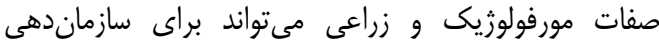

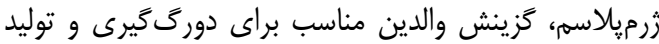

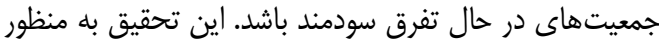

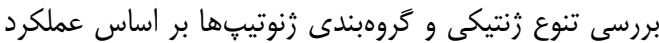

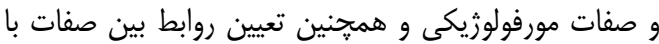

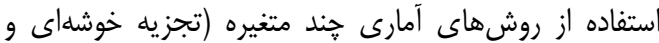
تجزيه به عامل ها) انجام شد.

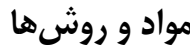

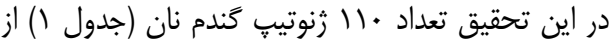

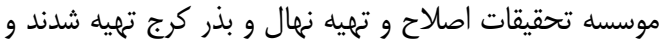

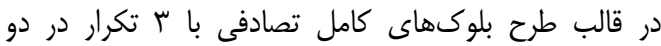

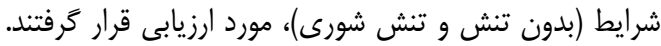

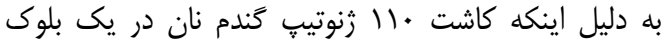

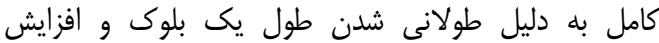

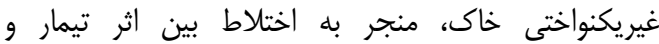

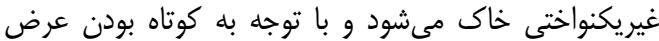

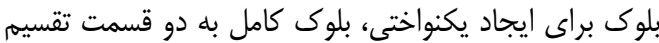

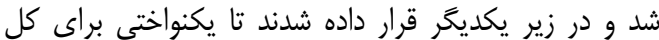

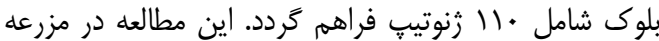

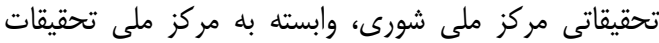

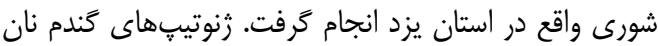

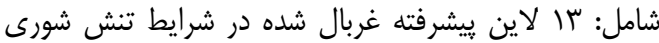

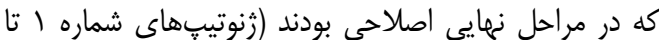

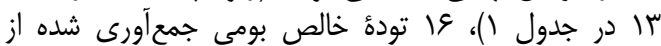

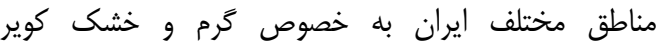

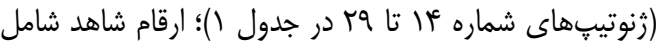

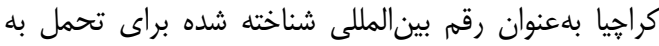

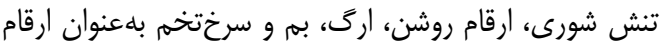

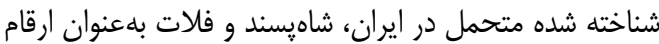

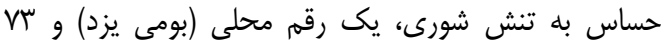

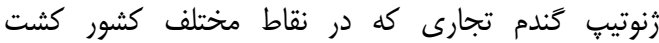

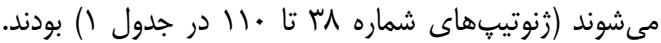

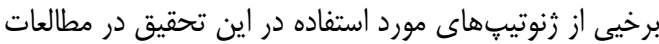

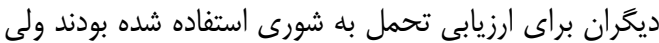

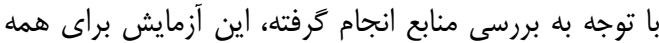

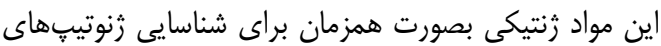

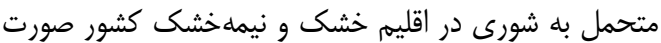

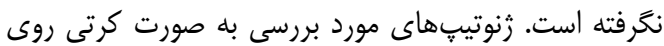




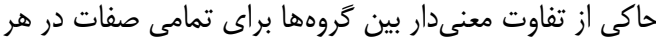

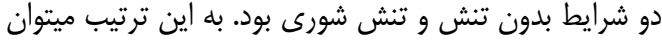

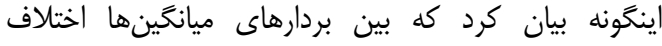

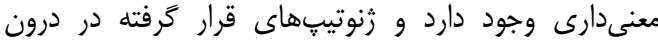

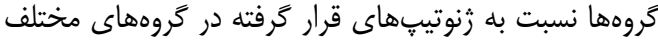

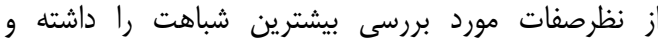

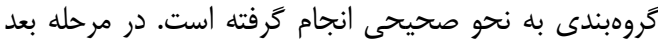

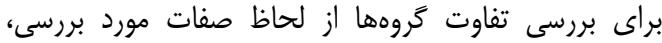

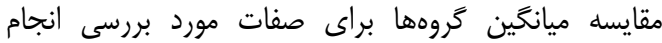

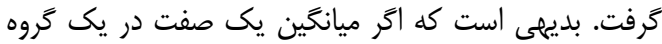

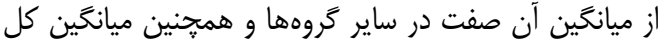

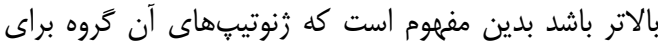

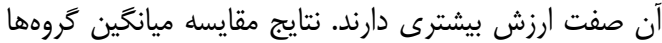

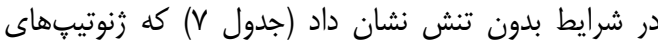

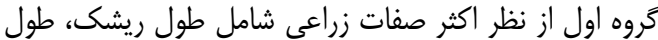

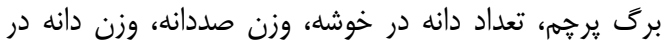

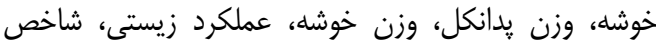

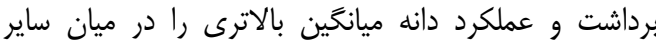

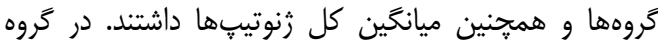

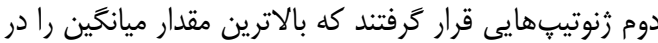

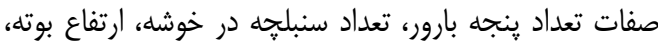

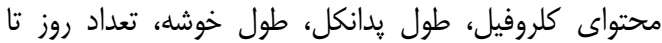

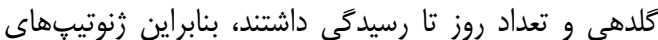

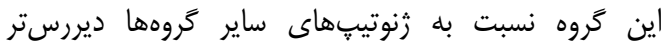

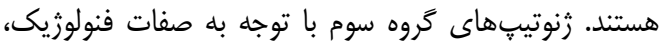

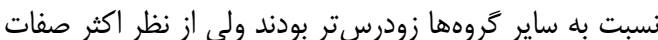

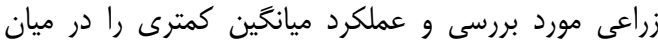

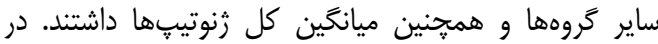

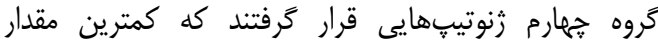

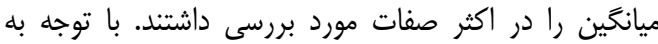

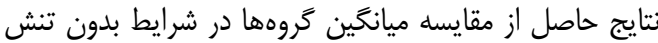

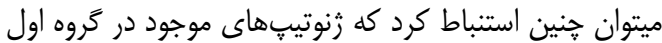

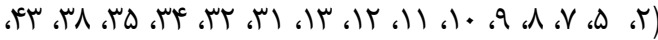

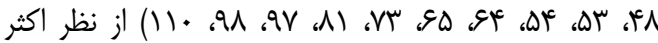

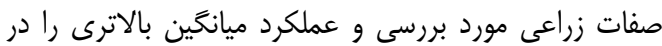

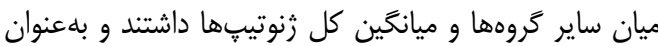

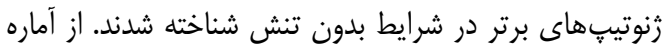
ماهالانوبيس براى تعيين فاصله كَروهها استفاده شد. نتانئ نتايج

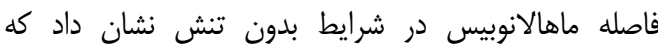

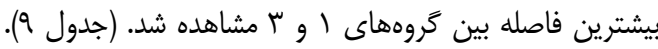

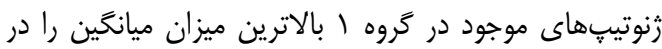

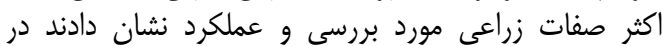

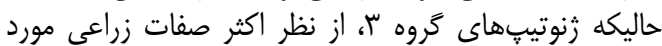

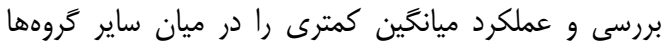

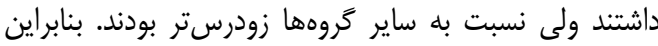

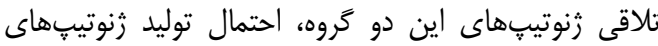

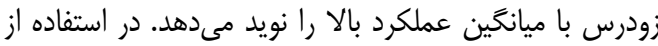

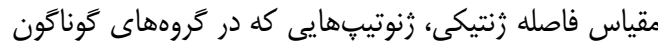

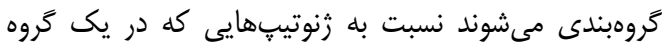

ينهانى از تجزيه به عاملها استفاده شد. براى استخراج

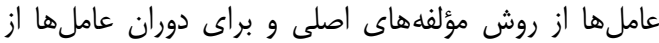

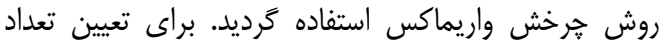

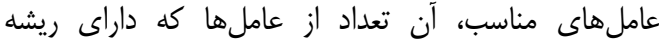

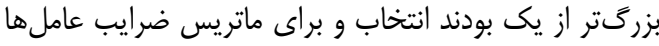

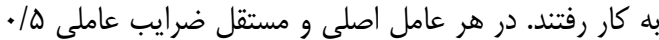

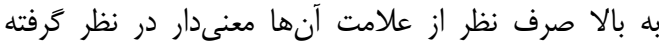

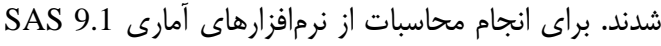

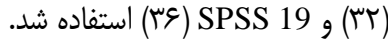

\section{تنتايج و بحث تجزيه خوشهاى تئي}

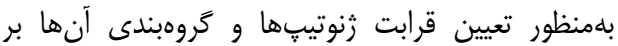

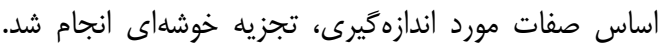

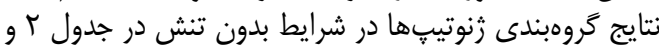

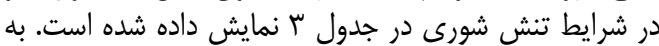

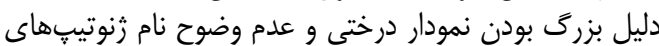

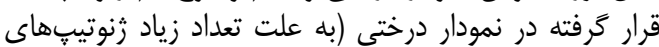

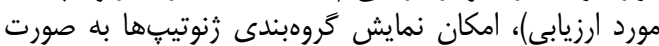

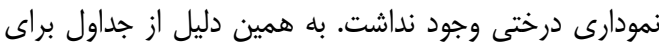

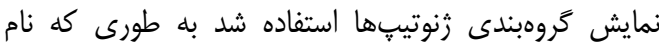

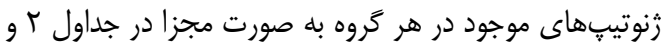

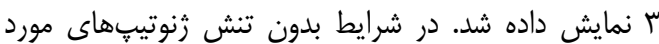

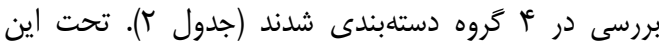

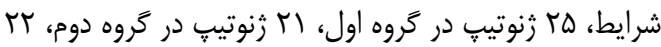

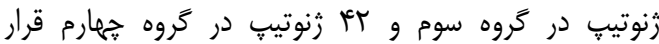

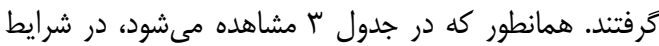

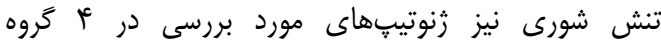

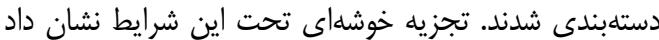

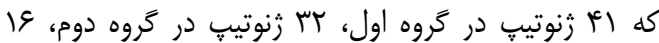

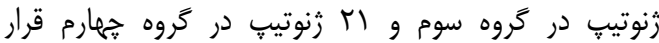

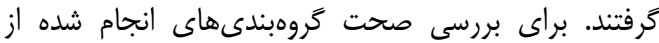

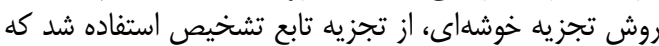

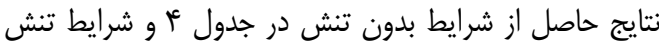

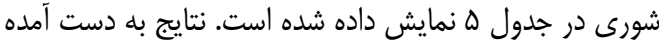

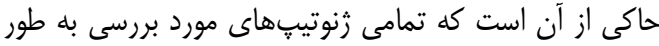

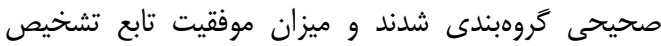

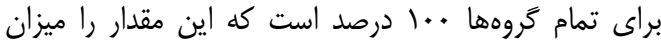

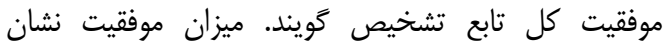

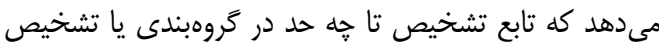

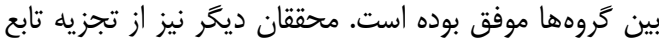

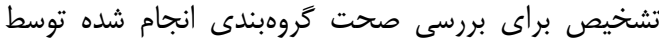

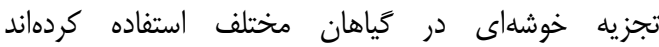

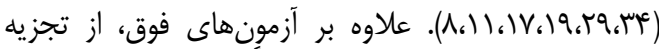

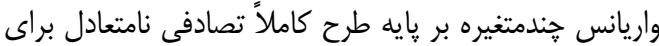

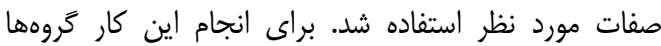

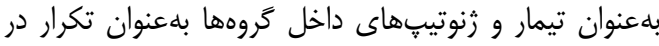

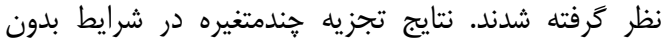
تنش و تنش شورى در جدول 9 نمايش داده شده است. نتايج 
تعداد دانه در خوشه، وزن صددانه، وزن دانه دانه در خوشه، وزن

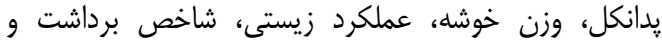

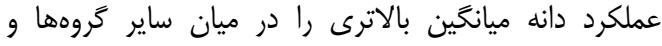

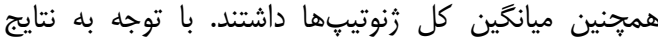

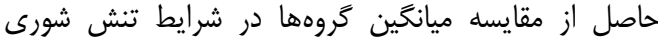

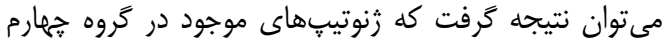

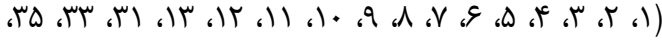

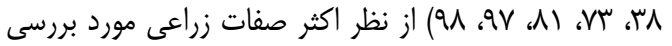

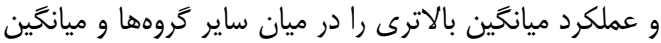

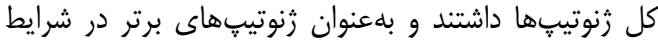

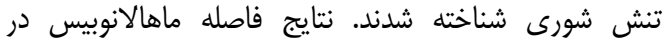
شرايط تنش شورى نشان داد كه بيشترين فاصله

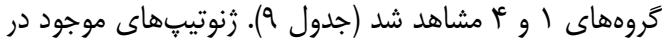

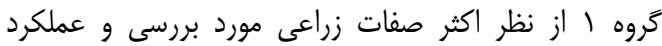

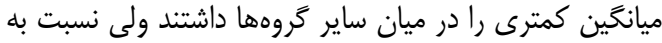

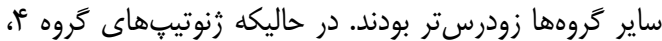

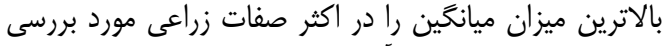

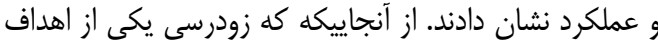

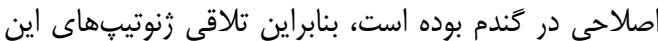

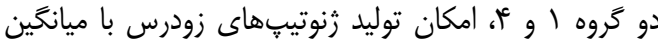
عملكرد بالا را در شرايط تنش شورى نويد مئد توند.
قرار مى گيرند، شباهت كمترى وجود دارد. در برنامههاى

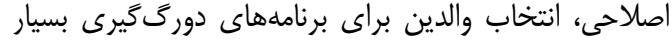

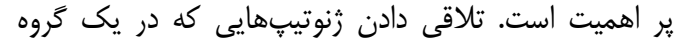

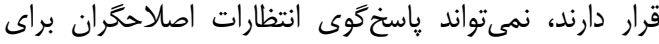

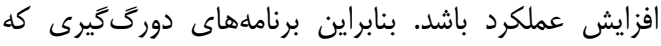

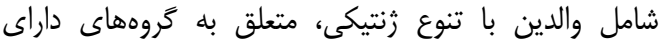

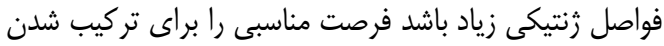

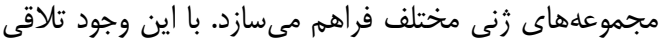

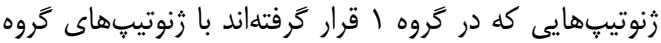

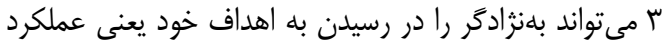

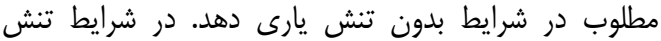

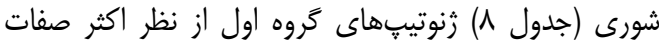

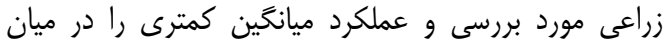

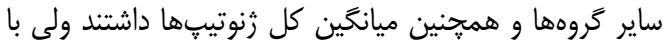

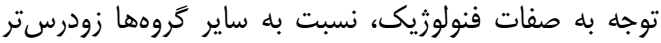

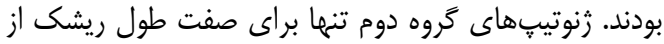

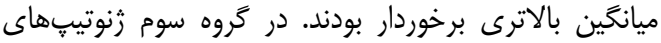

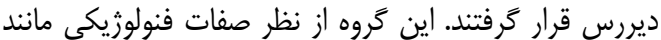

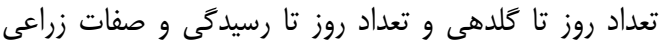

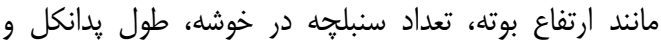

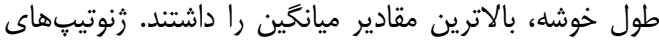

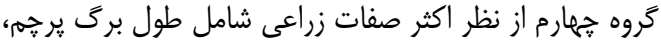

Table 1. The codes and name of wheat genotypes

جدول ا- كد و نام زنوتيبهاى كُندم

\begin{tabular}{|c|c|c|c|c|c|c|c|}
\hline شماره & نام رنوتيّب & شماره & نام زنوتيبٍ & شماره & نام رنوتيب & شماره & نام رنوتيٍ \\
\hline 1 & Salt18 & rq & بومى IV & $\Delta V$ & קناب & $\Lambda \Delta$ & MV17 \\
\hline r & Salt19 & r. & كراجيا & $\Delta \Lambda$ & داراب r & N & نويد \\
\hline r & Salt20 & ו & ارى & $\Delta q$ & دريا & $\Lambda V$ & نيكنزاد \\
\hline$f$ & Salt21 & rT & بم & s. & دز & $M$ & "اميد \\
\hline$\Delta$ & Salt22 & سז & روشن & 8) & DN11 & 19 & بارسى \\
\hline 8 & Salt23 & re & سرخ تخم & gr & كاسكوثن & 9. & بيشكام \\
\hline v & Salt24 & ro & رقم محلى يزد & ז4 & كاسيارد & १) & بيشتاز \\
\hline$\wedge$ & Salt25 & ع & شاهيسند & 94 & قدس & $q 5$ & رسول \\
\hline 9 & Salt26 & rV & فلات & 90 & كلستان & q & سبالن \\
\hline 1. & Salt27 & r & عدل & 98 & هامون & 98 & سردارى \\
\hline 11 & Salt28 & rq & افلاك & SV & هيرمند & 90 & سايسون \\
\hline ir & Salt29 & f. & اكبرى ل اك & 81 & اينيا & $q 9$ & سياهان \\
\hline זו & Salt30 & i) & آلاموت & 99 & كرج & १V & شهريار \\
\hline if & بومى I & Ft & البرز & v. & كرجr & 91 & شيراز \\
\hline 10 & بومى r & re & الوند & vi & كرجr & 99 & شيرودى \\
\hline 18 & بومى ץ & if & آرتا & vr & كاوه & $1 .$. & شعله \\
\hline IV & بومى F & $i \Delta$ & آروم & vr & كوير & 1.1 & سيستان \\
\hline 11 & بومى ه & is & اروند & $v^{c}$ & خزر ا & $1 . r$ & سيوند \\
\hline 19 & بومى 9 & iv & اترك & Va & كراشاهه & $1 . r$ & استار \\
\hline$r$. & بومى V V & is & آزادى & Ve & لاين A & 1.5 & طبسى \\
\hline r) & بومى 1 & 19 & آذر r & W & مهدوى & $1 \cdot \Delta$ & تجن \\
\hline Tt & بومى 9 & $\Delta$. & بهار & $\mathrm{vA}$ & مارون & 1.9 & توس \\
\hline זr & بومى .1 & Q) & بيات & vq & مرودشت & $1 \cdot V$ & ويريناك \\
\hline re & بومى IT & $\Delta T$ & باز & ᄉ. & ميهن & $1 \cdot 1$ & زاكرس \\
\hline TQ & بومى سا & QT & بككراس روشن & 1) & مغان & 1.9 & زارع \\
\hline rq & بومى If & $\Delta F$ & بككراس روشن زمستانه & Ar & 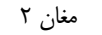 & 11. & زرين \\
\hline TV & بومى 10 بوا & $\Delta \Delta$ & بزوستايا & $\Lambda \mu$ & 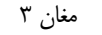 & & \\
\hline 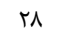 & | بومى 19 & $\Delta S$ & جمران & Af & مرواريد مريد & & \\
\hline
\end{tabular}


جدول r- تجزيه خوشهاى زنوتيبهاى گَندم با روش حداقل واريانس وارد بر اساس صفات اندازگيرى شده در شرايط بدون تنش Table 2. Cluster analysis of wheat genotypes by Ward's minimum variance based on measured traits in non-stress conditions

\begin{tabular}{|c|c|}
\hline كروه & شماره زنوتيبها \\
\hline 1 & 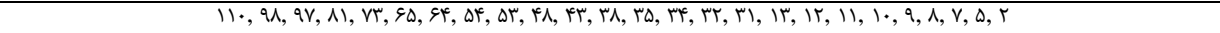 \\
\hline r & 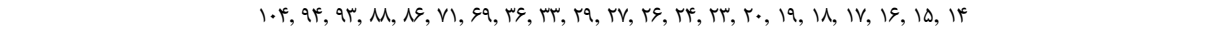 \\
\hline r & 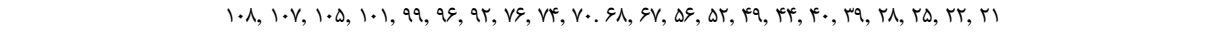 \\
\hline f & 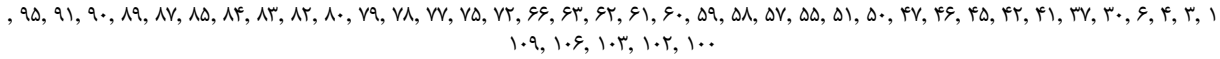 \\
\hline
\end{tabular}

جدول بـ- تجزيه خوشهاى زنوتيبهاى كَندم با روش حداقل واريانس وارد بر اساس صفات اندازمَيرى شده در شرايط تنش شورى Table 3. Cluster analysis of wheat genotypes by Ward's minimum variance based on measured traits in saline stress conditions

\begin{tabular}{|c|c|}
\hline شماره زنوتيبها & كرو \\
\hline 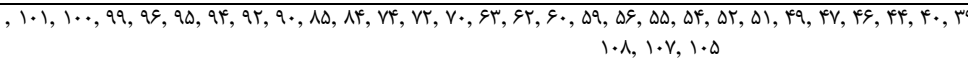 & 1 \\
\hline 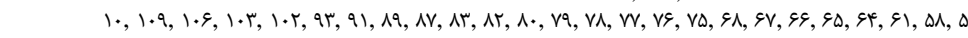 & r \\
\hline 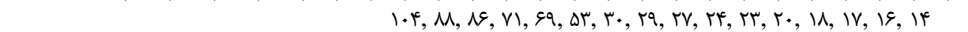 & r \\
\hline 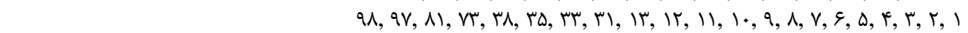 & f \\
\hline
\end{tabular}

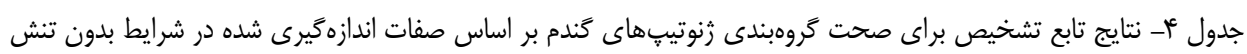

Table 4. The results of discriminant function for clustering validity of wheat genotypes based on measured traits in non-stress conditions

\begin{tabular}{|c|c|c|c|c|c|c|c|c|c|c|}
\hline \multirow{2}{*}{\multicolumn{2}{|c|}{ كل }} & \multicolumn{8}{|c|}{ كروههاى بيشيينى شده بر اساس تجزيه تابع تشخيص } & \multirow{3}{*}{ خروههاى حاصل از تجزيه } \\
\hline & & \multicolumn{2}{|c|}{ f } & \multicolumn{2}{|c|}{4} & \multicolumn{2}{|c|}{$\frac{1}{4}$} & \multicolumn{2}{|c|}{1} & \\
\hline درصد & تعداد & درصد & تعداد & درصد & تعداد & مرصد & تعداد & درصد & تعداد & \\
\hline $1 \cdots$ & ro & $\cdot$ & . & . & . & $\cdot$ & . & $1+$. & $r \Delta$ & 1 \\
\hline $1 \ldots$ & rI & . & . & . & . & $1 \ldots$ & rI & . & . & r \\
\hline $1 .$. & r & . & . & $1 \ldots$ & r & . & . & . & . & r \\
\hline $1 \ldots$ & et & $1 \ldots$ & et & . & . & . & . & . & . & f \\
\hline
\end{tabular}

جدول ه- نتايج تابع تشخيص براى صحت گروهبندى زنوتيِهاى گندم بر اساس صفات اندازميرى شده در شرايط تنش شورى

Table 5. The results of discriminant function for clustering validity of wheat genotypes based on measured traits in saline stress conditions

\begin{tabular}{|c|c|c|c|c|c|c|c|c|c|c|}
\hline \multirow{2}{*}{\multicolumn{2}{|c|}{ كل }} & \multicolumn{8}{|c|}{ كروههاى بيشبينى شده بر اساس تجزيه تابع تشخيص } & \multirow{3}{*}{ خروههاى حاصل از تجزيه } \\
\hline & & \multicolumn{2}{|c|}{ f } & \multicolumn{2}{|c|}{$r$} & \multicolumn{2}{|c|}{$r$} & \multicolumn{2}{|c|}{1} & \\
\hline درصد & تعداد & درصد & تعداد & درصد & تعداد & درصد & تعداد & درصد & تعداد & \\
\hline $1 \ldots$ & 41 & . & $\cdot$ & . & . & $\cdot$ & . & 1. & 41 & 1 \\
\hline $1 \ldots$ & rT & • & . & • & • & $1 \ldots$ & rr & • & • & r \\
\hline $1 .$. & 18 & . & . & $1 .$. & 19 & . & . & . & . & r \\
\hline $1 .$. & r) & $1 .$. & rI & . & . & . & . & . & . & r \\
\hline
\end{tabular}

جدول و- تجزيه واريانس كروهها بر اساس صفات اندازهكيرى شده در شرايط بدون تنش و تنش شورى

Table 6. Analysis of variance based on measured groups in non-stress and saline stress conditions

\begin{tabular}{|c|c|c|c|c|}
\hline \multicolumn{2}{|c|}{ شرايط تنش شورى } & \multicolumn{2}{|c|}{ شرايط بدون تنش } & \multirow[t]{2}{*}{ صفات } \\
\hline واريانس درون گروهى & واريانس بين گروهى & واريانس درون گروهى & واريانس بين گروهى & \\
\hline 1.9 & $r$ & 1.9 & $r$ & درجه آزادى \\
\hline 19/ & m & rN/Tr & $r \Delta I / \Delta T^{n+m}$ & ارتفاع بوته \\
\hline$r / M$ & $1 / 48^{\circ}$ & $r / \Lambda F$ & $N / T^{\top}$ & طول ريشك \\
\hline$r \Delta / \& \varnothing$ & $T \Delta F / T F^{m T}$ & TN/GD & FVV/Or & تعداد دانه در خوشه \\
\hline$I V / 9 V$ & $\mid V D / A T^{n+m}$ & TL/rq & $1 \cdot r /\left.9\right|^{\circ}$ & تعداد ينجه بارور \\
\hline $1 / 8$. & $\mid f / \| f^{m-T}$ & זس/ו & $r F / K F^{N-T}$ & تعداد سنبلحه در خوشه \\
\hline.$/ 9 \Delta$ & T/T & ع ع/1 & $F / \mathrm{VA}^{\circ}$ & طول برك يرجهم \\
\hline זו/. & $\cdot / \Delta r^{T T}$ & $\cdot / 4$. & $1 / \pi \Delta^{\pi m}$ & وزن صد دانه \\
\hline.$/ \Delta V$ & $1.194^{-\pi T}$ & $\cdot|\Lambda|$ & 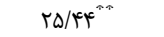 & وزن دانه در خوشه \\
\hline.$/ \cdot r$ & $\cdot / 4 \theta^{m \pi}$ & r & $\cdot / \Delta \mu^{\omega *}$ & وزن بدانكل \\
\hline •/ $/ \Delta$ & $W / Q \Lambda^{-\pi}$ & $1 / \cdot 1$ & $r g / V f^{m+}$ & وزن خوشه \\
\hline$|r / 9|$ & $1 \% \cdot / 98^{\pi m}$ & $1 . / 94$ & $1 \cdot r / \Delta \cdot{ }^{N \pi}$ & محتواى كلروفيل \\
\hline $1 / / 9 q$ & 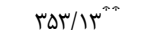 & $r \cdot|\Delta|$ & $9|r / q|^{n-m}$ & تعداد روز تا كلدهى \\
\hline q/Af & $r \varphi q / V \Lambda^{m *}$ & $q|q|$ & ए人१/५q" & تعداد روز تا رسيدگى \\
\hline $11 / \cdot v$ & $\| \varepsilon / T \varepsilon^{\pi \cdot r}$ & $\mid f / F \Delta$ & r & طول يدانكل \\
\hline عس/. & $1 / 90^{n-\pi}$ & $\cdot / \Delta \omega$ & $\Delta / r V^{m-\sigma}$ & طول خوشه \\
\hline rATA/VV & $\Lambda F V V V / \mu \Lambda^{* \pi}$ & 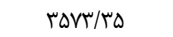 & $11 K \Phi V \cdot / 99^{\pi * T}$ & عملكرد \\
\hline$r V / / I$ & rQVEN/DV ${ }^{\top \pi}$ & VTr/DA & TrFeq/10 & عملكرد دانه \\
\hline $.1 \cdot 1$ &.$/ 19^{\pi T}$ & $.1 \cdot 1$ & $\cdot / \cdot e^{-\pi *}$ & شاخص برداشت \\
\hline
\end{tabular}


Table 7. The traits average in each group in non-stress conditions

\begin{tabular}{|c|c|c|c|c|c|}
\hline ميانگين كل & كروه جهارم & كروه سوم & كروه دوم & كروه اول & صفات \\
\hline 11. & FT & $\pi$ & rI & Ta & تعداد رنوتيبِ \\
\hline$\varepsilon^{4} /<q$ & $9 \cdot / 99^{\circ}$ & $\varepsilon 1 / 4 \varepsilon^{D}$ & $\varepsilon_{N} / \mu^{\mathrm{a}}$ & $90 / \wedge 9^{a}$ & ارتفاع وته \\
\hline$\Delta / \uparrow$. & D/rسab & $\mathrm{Q} / \Delta \mathrm{V}^{\mathrm{ab}}$ & $F / V Y^{D}$ & s/l $\mathrm{r}^{\mathrm{a}}$ & طول ريشك \\
\hline ro/qu & $\Gamma N / \Delta V^{\mathrm{a}}$ & $\Gamma \cdot / v \varepsilon^{D}$ & D & $r q / 1 q^{a}$ & تعداد دانه در خوشه \\
\hline Tr/VT & $\mathrm{r} / \cdot \mathrm{\Lambda}^{\mathrm{D}}$ & $\Gamma \mathrm{T} / 90^{\mathrm{D}}$ & r & r & تعداد ينجه بارور \\
\hline $19 / 1$. & $19 / \mathrm{HV}^{\mathrm{D}}$ & $\mid r / \Delta \gamma^{c}$ & $\mid V / \cdot r^{\mathrm{a}}$ & $19 / \pi 0^{\circ}$ & تعداد سنبلحه در خوشه \\
\hline $\mid r / \cdot \Delta$ & $11 / 9 \varphi^{D}$ & $\mid r / \cdot \Delta^{\mathrm{ab}}$ & $\mid r / r^{\mathrm{ab}}$ & $\mid r / \Delta \gamma^{\mathrm{a}}$ & طول بركَ يرجما \\
\hline$r / \pi r$ & $r / \cdot r^{\mathrm{DC}}$ & $r / \Lambda \gamma^{c}$ & $\Gamma / \Gamma \Delta^{\mathrm{ab}}$ & $r / q^{a}$ & وزن صد دانه \\
\hline$g / T F$ & $s / \mathrm{rV}^{\mathrm{D}}$ & $F / N \mathcal{F}^{\mathrm{C}}$ & $s / \cdot V^{0}$ & $V / \mathrm{rq}^{\mathrm{a}}$ & وزن دانه در خوشه \\
\hline 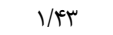 & $1 / r^{D}$ & $1 / 4^{c}$ & $1 / \Delta F^{a}$ & $1 / \Delta \mathrm{r}^{\mathrm{a}}$ & وزين بدانكل \\
\hline$V / r q$ & $V / F \Lambda^{D}$ & $\Delta / \Delta q^{c}$ & $V / 48^{D}$ & $N / q F^{a}$ & وزن خوشه \\
\hline$\Delta T / \cdot V$ & $\Delta) / r^{D}$ & $\left(99 / V^{0}\right.$ & $\Delta F / T^{a}$ & $\Delta r / \Delta V^{a}$ & محتواى كلروفيل \\
\hline $1 f \cdot / \pi$ & 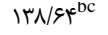 & $\mid r \varepsilon / \Delta \Lambda^{c}$ & 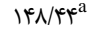 & ( & تعداد روز تا كلدهى \\
\hline$|V| r A$ & $199 / 19^{c}$ & $\mid 9 \mathrm{~V} / \cdot 9^{\mathrm{a}}$ & $\mid V \varepsilon / A v^{a}$ & $\mid V T / 4 .^{\circ}$ & تعداد روز تا رسيدگى \\
\hline & $r \cdot / \Lambda^{\mathrm{D}}$ & $r q / 90^{\circ}$ & $r 8 / 8 \Lambda^{a}$ & $T I / T \Delta^{D}$ & طول پيدانكل \\
\hline N/9D & $N / N^{c}$ & $\Lambda / v \varepsilon^{c}$ & $q / r \Delta^{a}$ & $q / 1 .{ }^{a b}$ & طول خوشه \\
\hline TAT/AT & $r \Delta I / \mu^{c}$ & $r \mu q / q^{c}$ & $r q \Delta / V \varepsilon^{D}$ & $r v \varepsilon / I V^{a}$ & عملكرد \\
\hline$Q N / \Delta T$ & १)/Vब ${ }^{\mathrm{D}}$ & $V W / \varphi^{k^{c}}$ & $M / \kappa \mu^{D}$ & $\mid F r / \cdot \wedge^{a}$ & عملكرد دانه \\
\hline.$/ \%$ &.$/ \Lambda^{\mathrm{a}}$ & . &.$/ \cdot^{\mathrm{D}}$ &.$/ \mu^{\mathrm{a}}$ & شاخص برداشت \\
\hline
\end{tabular}

Table 8. The traits average in each group in saline stress conditions

\begin{tabular}{|c|c|c|c|c|c|}
\hline ميانكين كل & كروه يهارم & كروه سوم & كروه دوم & كروهاول & صفات \\
\hline 11. & TI & 19 & rt & fi & تعداد رنوتيبي \\
\hline DI/TV & $\Delta F / F q^{a}$ & $\Delta \& 1 \cdot q^{\mathrm{a}}$ & $\omega \cdot / \mathrm{vq}^{\mathrm{D}}$ & 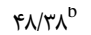 & ارتفاع بوته \\
\hline$r / 9 \Lambda$ & $\Delta / .9^{\mathrm{a}}$ & $\Gamma / 9 \cdot{ }^{0}$ & $\Delta / T^{\mathrm{a}}$ & $r / r \gamma^{a b}$ & طول ريشك \\
\hline ta/k & $\mathrm{r} / / \kappa^{\mathrm{a}}$ & ( & $r I / r F^{a}$ & $r \omega / \& \Lambda^{c}$ & تعداد دانه در خوشه \\
\hline TY/TD & $\Gamma \Delta / q \omega^{\mathrm{a}}$ & $T r / q F^{D}$ & $19 / \Delta \mathcal{F}^{c}$ & $T r / / V^{D}$ & تعداد ينجه بارور \\
\hline$|F / \Delta|$ & $|F / N|^{D}$ & $10 / v q^{\mathrm{a}}$ & $\mid F / \Delta D^{\mathrm{DC}}$ & $\mid r / A \gamma^{c}$ & تعداد سنبلجه در خوشه \\
\hline Q/АF & $1 . / 1 \Lambda^{\mathrm{a}}$ & $9 / \wedge 1^{a b}$ & $1.110^{\mathrm{a}}$ & $9 / \uparrow \Lambda^{D}$ & طول برى يرجم \\
\hline$r / \notin q$ & $r / V)^{a}$ & $\Gamma / \omega \cdot{ }^{0}$ & $r / \notin V^{D}$ & $\Gamma / \mu^{D}$ & وزن صد دانه \\
\hline$r / 9 \Delta$ & $\Delta / \mathrm{rq}^{\mathrm{a}}$ & $r / \% .^{\circ}$ & $f / 9 q^{a b}$ & $r / .^{c}$ & وزن دانه در خوشه \\
\hline $1 /$. & $1 / r \omega^{a}$ & $1 / r^{\mathrm{a}}$ & $1 / \cdot \Lambda^{0}$ &.$/ 9 \Lambda^{\mathrm{c}}$ & وزن بدانكل \\
\hline D/DF & $s / \mu^{a}$ & $\Delta / \mathrm{r} \Lambda^{0}$ & $9 / \cdot e^{\mathrm{DC}}$ & $r / 9 \varphi^{\mathrm{C}}$ & وزن خوشه \\
\hline $\mathrm{FV} / \cdot 1$ & $\Delta \cdot / \Delta \gamma^{\mathrm{a}}$ & $\left\lceil N / q^{\varphi^{a}}\right.$ & $\Gamma \Delta / \cdot r^{D}$ & $\leftarrow \varepsilon / \Delta F^{D}$ & محتواي كلروفيل \\
\hline קצ/אזו & $\mid T V / A F^{D}$ & $|\notin q| .{ }^{a}$ & $1 \mathrm{rV} / \mathrm{Q} \Lambda^{\mathrm{D}}$ & $\mid r g / 4 \cdot{ }^{D}$ & تعداد روز تا كلدهى \\
\hline $19 N / 19$ & $19 N / 9 Y^{D}$ & $\mid V E / \mu^{a} \Lambda^{a}$ & $19 q / q \mu^{c}$ & $\mid \varepsilon q / T \Delta^{\mathrm{C}}$ & تعداد روز تا رسيدگى \\
\hline$r \mu / q$. & $T T^{\prime} / Y^{D}$ & $\mathrm{rV} / A \mathrm{~V}^{\mathrm{a}}$ & $r r / \Lambda \cdot{ }^{D}$ & $r r / \wedge \mu^{D}$ & طول بدانكل \\
\hline V/a9 & $N / \backslash 9^{\mathrm{a}}$ & N/F & $N / \Lambda^{\mathrm{a}}$ & $V / \subset q^{D}$ & طول خوشه \\
\hline$r . . / R$ & $r \Lambda \Delta / 1 F^{\mathrm{a}}$ & $r \pi \cdot / Q 1^{D}$ & $\mid \Lambda \Delta / v e^{c}$ & $\mid \Delta \Delta / V g^{a}$ & ع علكSد \\
\hline$s \mathrm{~V} / \cdot \mathrm{V}$ & $\mid r \Delta / \cdot \mathrm{ra}^{\mathrm{a}}$ & $\Delta F / V r^{D}$ & $\Delta q / / \varphi^{D}$ & 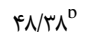 & عملكر د دانه \\
\hline . $/{ }^{\prime} \Delta$ & $\cdot / \mathrm{q}^{\mathrm{a}}$ &.$/ r \omega^{c}$ & 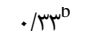 & ( ) & شاخص برداشت \\
\hline
\end{tabular}

جدول q- فواصل ماهالانوبيس بين گروهها در شرايط در شرايط بدون تنش (يايين قطر) و تنش شورى (بالاى قطر). Table 9. Mahalanobis distances between groups in non-stress (above diameter) and salinity stress (down diameter) conditions

\begin{tabular}{|c|c|c|c|c|}
\hline F & $r$ & $r$ & 1 & ك \\
\hline 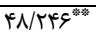 & $|Q / \bar{Q} \Delta|^{6 / 2}$ & $19 / 4 \cdot 1^{232}$ & & 1 \\
\hline$r V /\left.\Psi\right|^{* * *}$ & $\mid w / 4 \gamma * *$ & & $|V / \mu| y^{\text {stwe }}$ & r \\
\hline 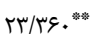 & & $r \Delta / \Lambda \subset \varepsilon^{*: B}$ & 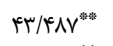 & r \\
\hline & $1 Q / A) \Lambda^{* * *}$ & $r \cdot \mid q \wedge 0^{* * B}$ & 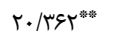 & r \\
\hline
\end{tabular}


توجيه نمود، داراى ضرايب بزرى و مثبت براى صفات

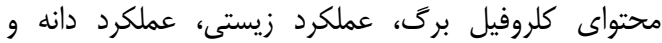

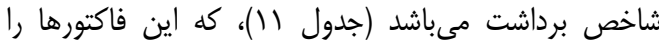

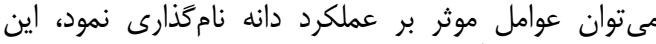

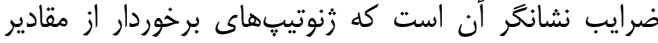

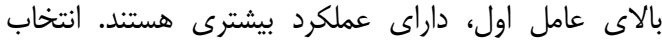

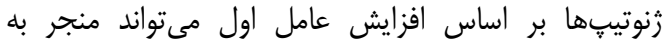
افزايش عملكرد در شرايط تنش شورى ائس در جمعيت مورد مطالعه گردد.

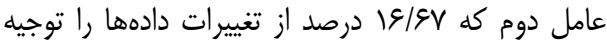

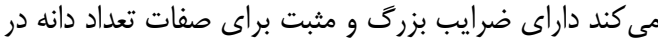

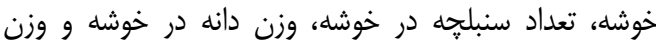

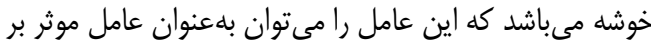

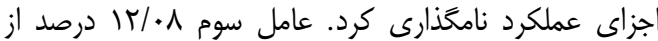

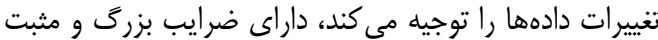

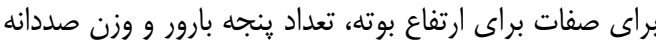

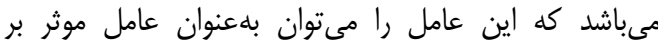

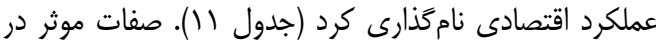

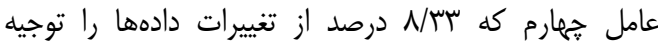

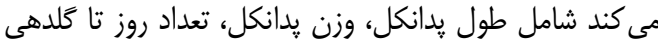

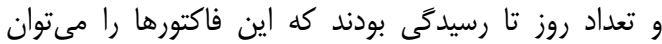

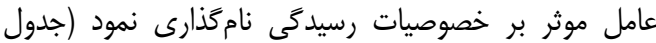

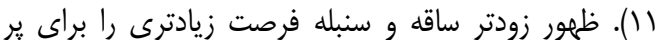

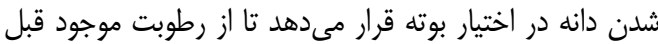

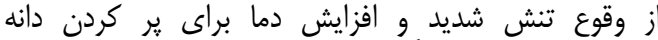

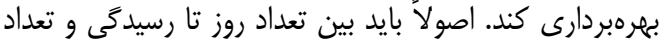

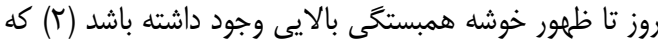

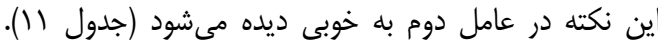

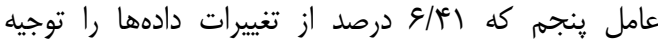

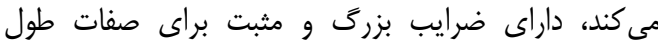

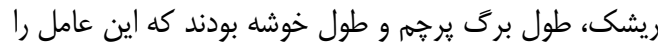

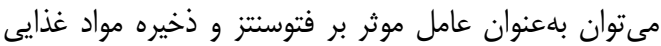

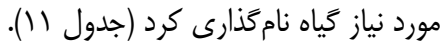

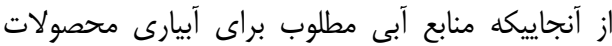

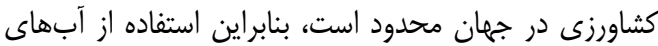

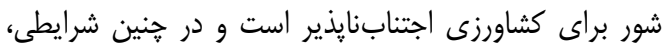

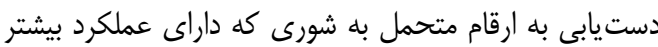

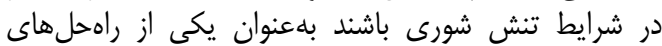

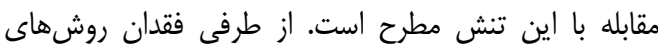

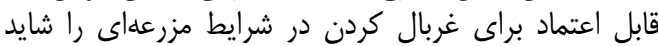

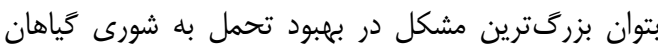

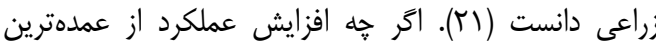

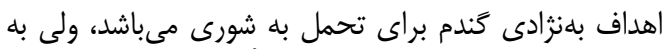

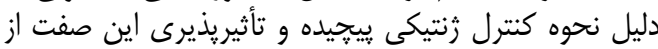

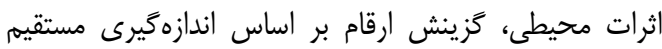

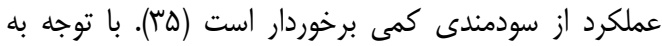

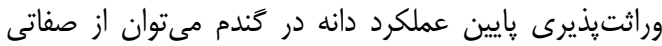

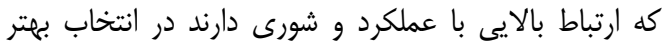

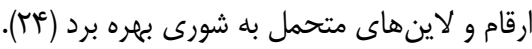

تجزيه به عاملها

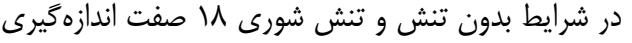

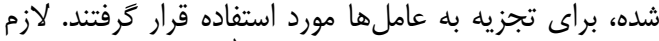

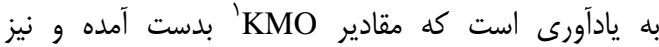

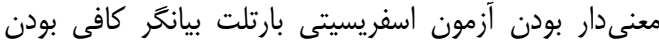

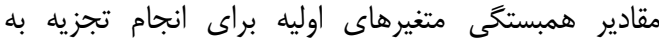

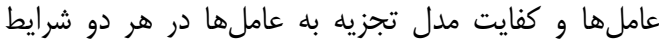

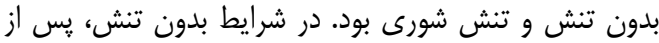

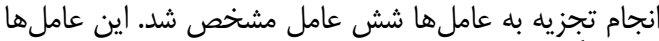

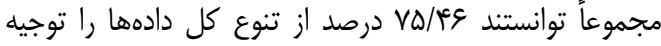

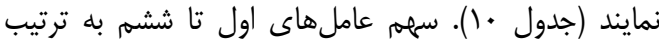
برابر كاود/

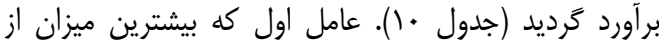

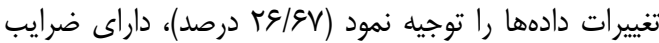

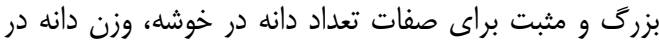

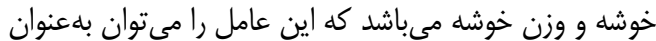

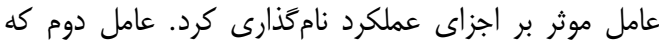

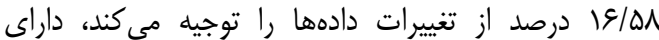

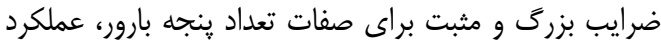

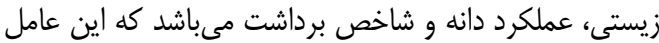

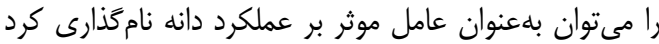

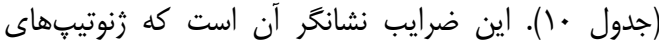

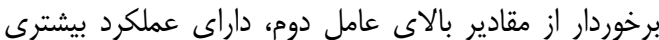

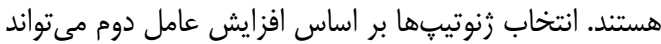

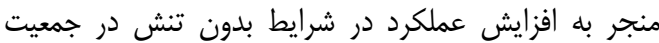

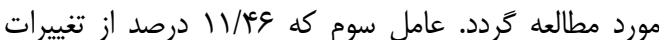

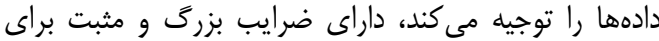

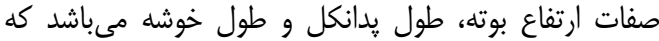

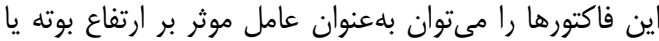

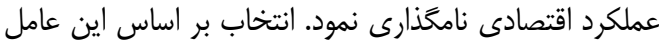

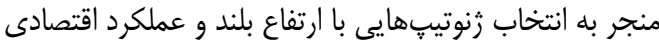

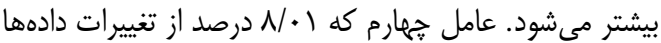

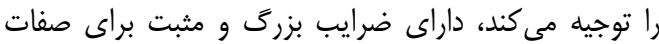

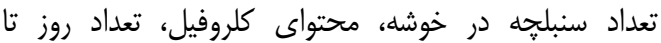

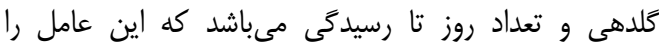

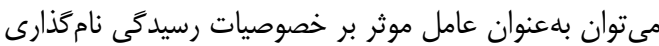

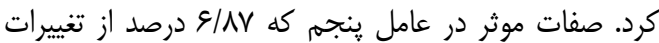

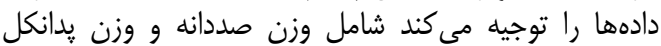

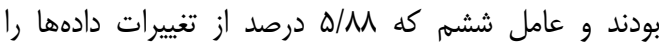

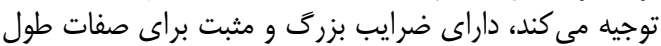

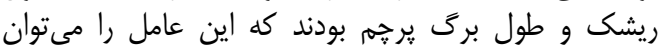

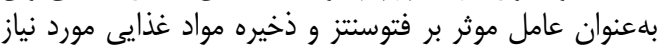

كياه نام كذارى كرد (جدول • (1) در شرايط تنش شورى، يس إن از انجام تجزيه به به عامله

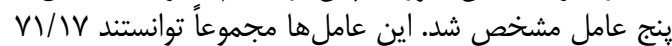

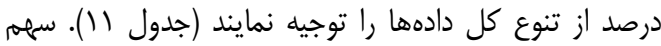

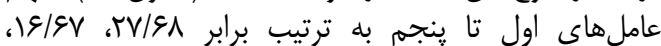

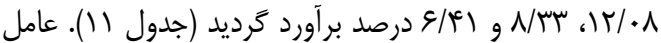

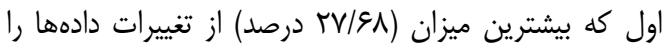


جدول • ا- ضرايب عاملى در تجزيه به عامل ها به روش مؤلفههاى اصلى و دوران واريماكس در شرايط بدون تنش در گندم Table 10. Factor coefficients in factor analysis using principal components and varimax rotation under non-stress

\begin{tabular}{|c|c|c|c|c|c|c|}
\hline عامل ششم & عامل ينجهم & عامل جهارم & 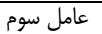 & عامل دوم & عامل اول & صفات \\
\hline$\cdot / \cdot 4^{4}$ & . &.$/ 14$ &.$/ 19$ &.$/ 1 \wedge$ &.$- / \cdot 1$ & ارتفاع بوته \\
\hline$\cdot / V T$ & $-.1 \cdot 0$ & זו/. - & $-\overline{-* / 1 F}$ & $.1 \cdot v$ &.$- / .1$ & طول ريشك \\
\hline$\overline{\cdot / \cdot \Lambda}$ & $-/ \mu r$ & $.1 \cdot 1$ & $-.1 \cdot 1$ &.$- / \cdot r$ & $\cdot / \mathrm{AV}$ & تعداد دانه در خوشه \\
\hline-.1 .0 & .1 .9 & .1 .9 &.$/ 1 f$ & $\cdot / \mathrm{N} /$ &.$- \cdot 19$ & تعداد ينجه بارور \\
\hline$-\cdot / 19$ &.$- / 19$ & $\cdot / \Delta V$ & ./TA &.$- \cdot \cdot v$ &.$/ 0$. & تعداد سنبلجه در خوشه \\
\hline .194 & . & $\overline{-. / . \mu}$ & •/tt & $-\cdot / \cdot V$ &.- .1 .1 & طول برى يرجهم \\
\hline$\overline{. / \cdot \varphi^{4}}(-1)$ & . & $.1 .9-$ & $.1 \cdot 0$ & $\cdot / T \Lambda$ &.$/ \cdot v$ & وزن صد دانه \\
\hline-.1 .4 & $\overline{. / r v}$ & $\cdot / \cdot \mu$ & .1 .9 & ./4t & . /At & وزن دانه در خوشه \\
\hline ع & - /at & . & •/re & .1 .9 & $\overline{\cdot / r}$ & وزن بد بدانكل \\
\hline $.1 \cdot 1$ & 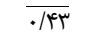 & .1 .9 & $\cdot 1 \cdot \mathrm{V}$ & .119 &.$/ v 9$ & وزن خوشه \\
\hline$-\cdot / 1 \mu$ & $-.1 \cdot 0$ &.$/ 9 \mathrm{~V}$ & $\cdot 1 \cdot 0$ & $\cdot / 4$. & $\overline{. \pi}$ & محتواى كلروفيل \\
\hline-.1 .9 & -.1 .5 & $\overline{\cdot / M}$ &.$八$ & $-\cdot / 1 f$ & $-.1 \cdot 1$ & تعداد روز تا كلدهى \\
\hline 正 & ./4q & $\overline{\cdot / \Lambda \varnothing}$ & $\cdot|r|$ & $.1 \cdot 0$ & $\cdot 1 \cdot 1$ & تعداد روز تا رسيدگى \\
\hline תוr &.$/ 4$. & $\overline{.} /{ }^{\top}$ & $\cdot|\lambda|$ &.$/ \cdot r$ & $-0 / 1$ & طول بِدانكل \\
\hline$\cdot / 4 V$ & ו וץ/.• & $\cdot 1 \cdot 1$ &.$/ 9 \mu$ & $\cdot 1$ & ع & طول خوشه \\
\hline 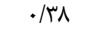 &.$/ 19$ & $\cdot / r \mid$ & $\cdot / \mu$ &.$|9|$ & 的 & عملكرد \\
\hline$\cdot / \mathrm{V}$ & $\cdot / r$. & $-.1 \cdot 0$ & $\cdot / \cdot v$ & $\overline{\cdot / \lambda \mid}$ & •/rq & 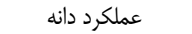 \\
\hline - & .1 .9 & $-* / \mu V$ & 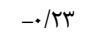 &.$/ \Delta V$ & . & شاخص برداشت \\
\hline $1 / .9$ & $1 / \Gamma^{4}$ & $1 / 4 F^{2}$ & $r / .9$ & $\overline{\Gamma / Q \Lambda}$ & $\varphi / \Lambda$. & مقادير ويثه \\
\hline$\Delta / \mathcal{M}$ & S/AV & $N \cdot 1$ & $11 / 49$ & $\mid \varepsilon / \Delta \Lambda$ & re/9V & مقدار ويثه ٪ \\
\hline$V \Delta / \leftarrow \&$ & $99 / 09$ & $G T / N T$ & $\Delta F / V I$ & $F / T \Delta$ & rE/ $/ 9 \mathrm{~V}$ & سهم تجمعى \\
\hline
\end{tabular}

جدول لا - ضرايب عاملى در تجزيه به عاملها به روش مؤلفههاى اصلى و دوران واريماكس در شرايط تنش شورى در گَندم Table 11. Factor coefficients in factor analysis using principal components and varimax rotation under saline stress

\begin{tabular}{|c|c|c|c|c|c|}
\hline عامل ينجم & عامل جهارم & عامل سوم & عامل دوم & عامل اول & صفات \\
\hline .148 & T &.$/ 95$ &.$- / .4$ & $-\cdot / .4$ & ارتفاع بوته \\
\hline.$/ \Delta V$ & - - D & $-\cdot / T V$ &.$- / 1 \pi$ & עזא/. & طول ريشك \\
\hline$\cdot / \cdot \mathrm{V}$ & $\%$ & 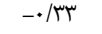 & $\cdot / A F$ &.$/ 1 f$ & تعداد دانه در خوشه \\
\hline.$- / 1 f$ & كt &. & .1 .1 & $\cdot / 4 V$ & تعداد ينجه بارور \\
\hline$\%$ &.$/ 49$ & $\overline{-. \cdot T \mid}$ &.$/ 9$. & $-\cdot / / V$ & تعداد سنبلجهه در خوشه \\
\hline$\cdot / \mathrm{V} \mid$ & $-\cdot|r|$ & 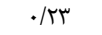 &.$/ 11$ &.$- / .0$ & طول برى بِ رجم \\
\hline$\cdot / \pi$ & ש & $\cdot|A|$ &.$/ 11$ &.$/ .4$ & وزن صد دانه \\
\hline.$/ .1$ &.$- / .4$ &.$/ \Gamma \Lambda$ & . /At & $\cdot / \cdot V$ & وزن دانه در خوشه \\
\hline$\cdot / \mu r$ & .19. & 年 & $\cdot / \mu 1$ &.$/ \mathrm{V}$ & وزن بدانكل \\
\hline.$/ 10$ & $.1 \cdot \Delta$ & r & .1199 &.$/ 11$ & وزن خوشه \\
\hline$-\cdot / T V$ & $\cdot / \mathrm{V}$ & . / & $-\cdot / \cdot r$ & .191 & محتواى كلروفيل \\
\hline. & $\cdot / \mathrm{vq}$ &.$- / 19$ &.$/ .4$ & $-\cdot / \pi T$ & تعداد روز تا كلدهى \\
\hline$\cdot 11$ &.$/ A T$ & 西 & $\cdot 1 \cdot 1$ & $-\cdot / \pi r$ & تعداد روز تا رسيدگى \\
\hline.$/ 19$ & $.10 \Lambda$ & $\cdot / \Delta \Delta$ & $-. \cdot / \cdot r$ & $-\cdot / \pi f$ & طول يدانكل \\
\hline.$/ V \Delta$ &.$/ \mu$. & -.1 .1 &.$/ 1 T$ &.$- / .4$ & طول خوشه \\
\hline$\cdot / \pi f$ & . AT & $\cdot / \mu r$ & $\cdot / 4 q$ & $.10 \Lambda$ & عملكرد \\
\hline .1 .9 & $\cdot / 1 r$ & & זr/. &.$/ \mathrm{Vq}$ & عملكرد دانه \\
\hline.$- / 1 T$ &.$- / 4 V$ & -.0 .4 & $.1 \cdot 0$ & $\cdot / \mathrm{VQ}$ & شاخص برداشت \\
\hline $1 / 10$ & $1 / \Delta$. & $T / I V$ & $r / \cdot \cdot$ & $\overline{F / Q \Lambda}$ & مقادير ويزه \\
\hline $8 / 41$ & שז/N & $\mid r / \cdot 1$ & $19 / 9 \mathrm{~V}$ & TV/צA & مقدار ويثه \% \\
\hline$V / / V$ & s广/va & $\Delta S / A T$ & $\mu \in / \mu \Delta$ & TV/FA & سهم تجمعى \\
\hline
\end{tabular}

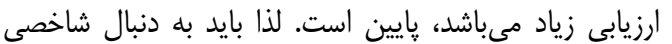

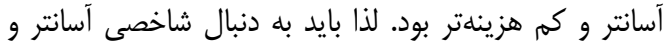
كم هزينهتر بود. در سالهاى اخير تعيين محتواى نسبى كلروفيل با استفاده

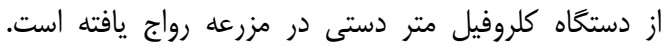

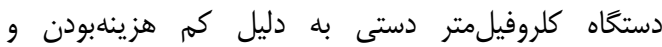

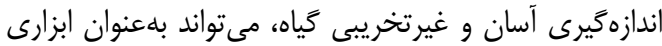

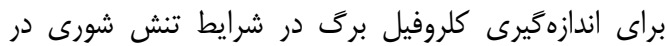

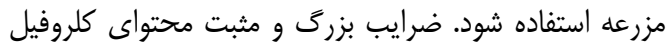

نتايج تجزيه به عاملها در شرايط تنش شورى مدات نشاندهنده ارتباط مثبت صفات محتواى كائ كلروفيل، عملكرد

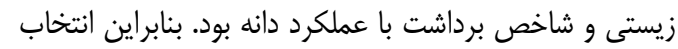

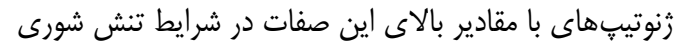

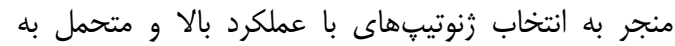

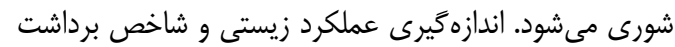

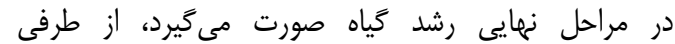

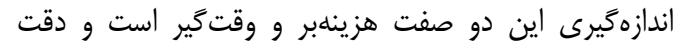

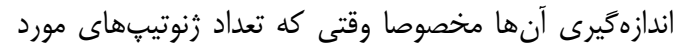


اين كاهش محتواى كلروفيل را تعديل كنند. بنابراين محتواى باى براي

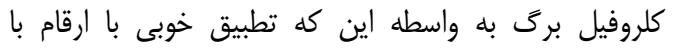

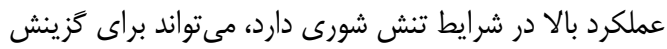

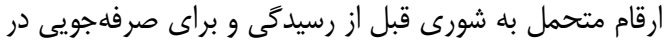

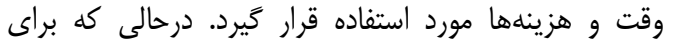

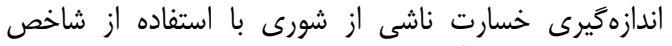

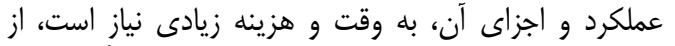

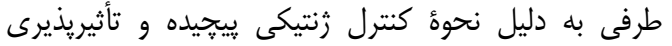

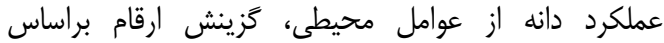

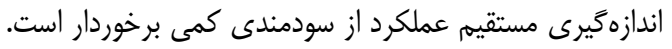

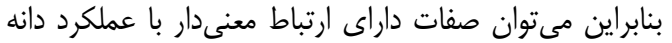

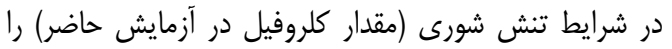

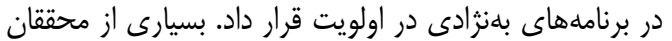

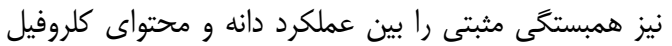

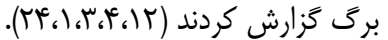

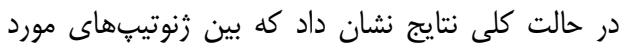

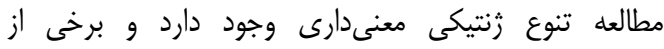

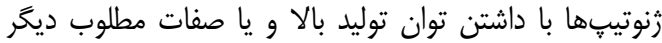

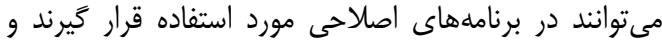

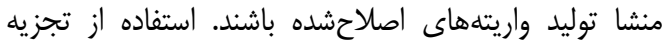

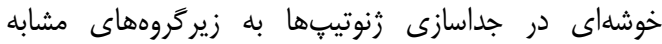

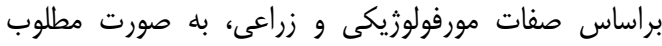

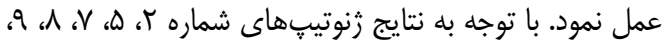

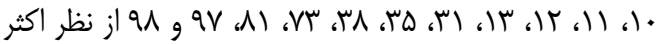

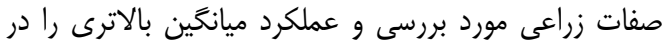

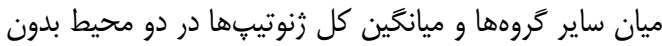

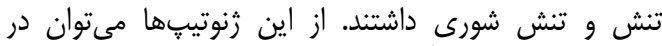

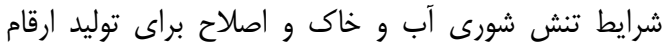

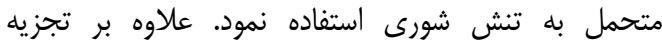

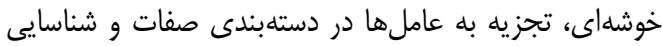

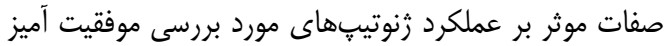

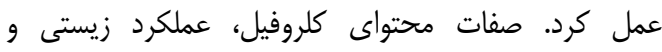

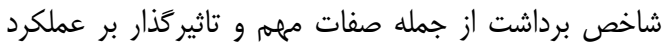

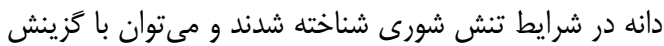

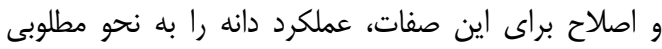

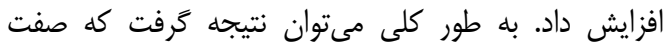

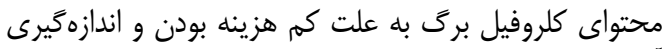

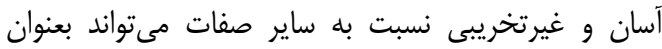

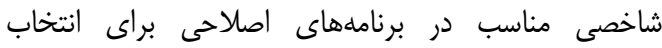

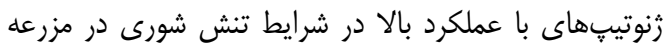

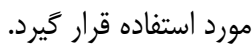

برگ و عملكرد دانه در عامل اول (جدول (1) نشاندهنده

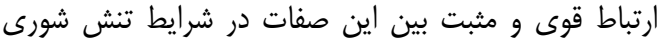

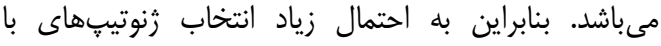

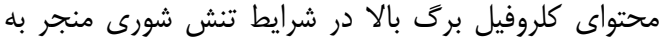

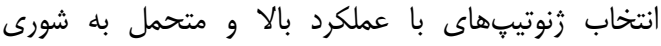

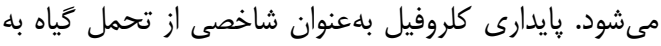

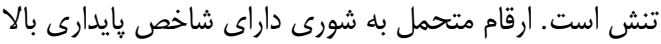

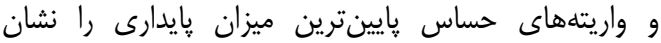

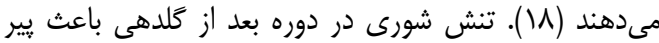

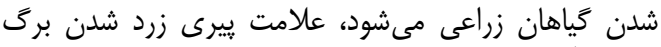

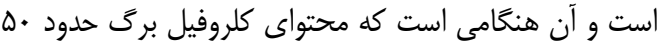

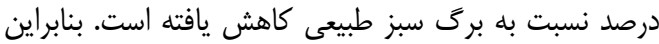

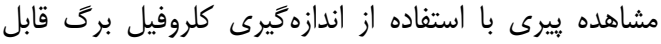

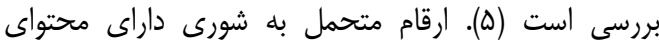

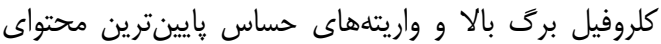

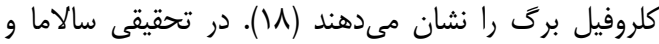

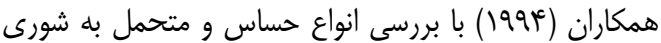

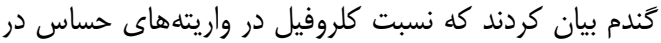

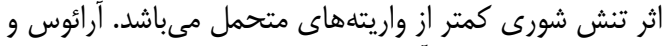

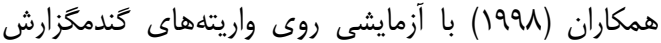

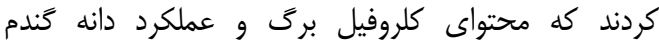

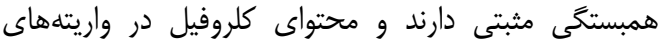

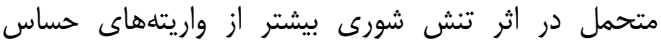

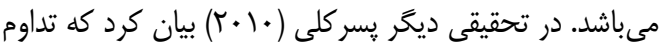

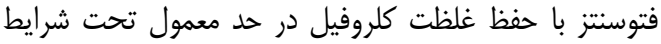

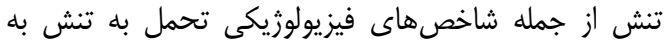

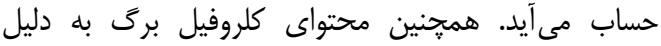

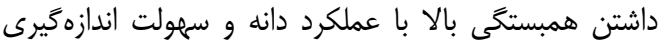

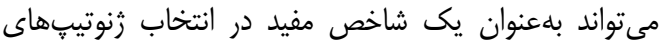

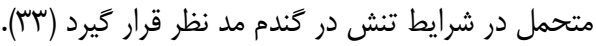

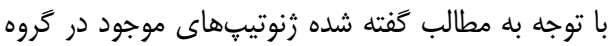

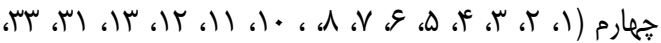

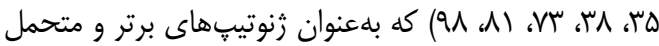

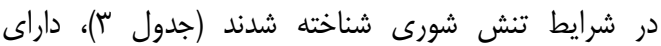

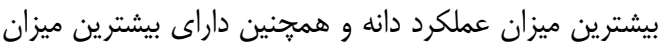

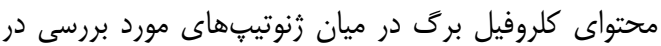

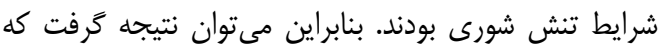

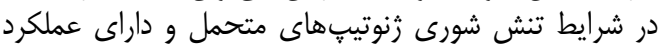

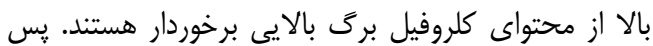

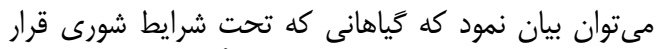

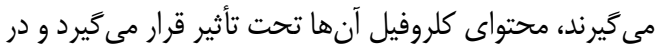

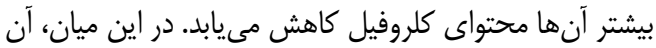

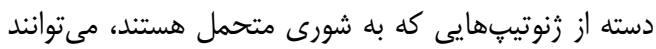


1. Araus, J., T. Amaro, J. Voltas, H. Nakkoul and M. Nachit 1998 . Chlorophyll fluorescence selection criterion for grain yield in durum wheat under Mediterranean conditions. Field Crop Research, 55: 209-223.

2. Blum, A. 1988. Plant Breeding for Stress environments. CRC Press Florida, 212 pp.

3. Boggs, J.L., T. Tsegaye, T.L. Coleman, K. Reddy and A. Fahsi. 2003. Relationship between hyperspectral reflectance, soil nitrate-nitrogen, cotton leaf chlorophyll, and cotton yield: a step toward precision agriculture. Journal of Sustainable Agriculture, 22: 5-16.

4. Bronson, K.F., T.T. Chua, J. Booker, J.W. Keeling and R.J. Lascano. 2003. In-season nitrogen status sensing in irrigated cotton. Soil Science Society of America Journal, 67: 1439-1448.

5. Cha, K.W., Y.J. Lee, H.J. Koh, B.M. Lee, Y.W. Nam and N.C. Paek. 2002. Isolation, characterization, and mapping of the stay green mutant in rice. Theoretical and Applied Genetics, 104: 526-532.

6. Colmer, T.D., T.J. Flowers and R. Munns. 2006. Use of wild relatives to improve salt tolerance in wheat. Journal of Experimental Botany, 57: 1059-1078.

7. El-Hendawy, S.E., Y. Hu, G.M. Yakout, A.M. Awad, S.E. Hafiz and U. Schmidhalter. 2005. Evaluating salt tolerance of wheat genotypes using multiple parameters. European Journal of Agronomy, 22: 243-253.

8. Hazegh-Jafari, P., S. Aharizad, S.A. Mohammadi, F. Noormand Moayyed and P. Behrooz. 2014. Grouping of alfalfa genotypes based on different characteristics using multivariate statistical analysis. Jornal of Crop Breeding, 13: 107-121 (In Persian).

9. Houshmand, S., A. Arzani, S.A.M. Maibody and M. Feizi. 2005. Evaluation of salt-tolerant genotypes of durum wheat derived from in vitro and field experiments. Field Crop Research, 91: 345-354.

10. Irvani, M., M. Soluki, A.M. Rezai, B. Syasar and S.H.A. Kuhkan. 2008. Investigating the diversity and relationship between agronomical traits and seed yield in barley advanced lines using factor analysis. Journal of Science and Technology of Agriculture and Natural Resourses, 45: 137-145 (In Persian)

11. Jaynes, D., T. Kaspar, T. Colvin and D. James. 2003. Cluster analysis of spatiotemporal corn yield patterns in an Iowa field. Agronmy Journal, 95: 574-586.

12. Kabanova, S. and M. Chaika. 2001. Correlation analysis of triticale morphology, chlorophyll content and productivity. Journal of Agronomy and Crop Science, 186: 281-285.

13. Keshavarznia, R., B. Mohammadi Nargesi and A.R. Abassi. 2013. Study of genetic diversity of common bean based on morphological traits under both normal and drought stress conditions. Iranian Journal of Field Crop Science, 44: 305-315 (In Persian).

14. Leilah, A. and S. Al-Khateeb. 2005. Statistical analysis of wheat yield under drought conditions. Journal of Arid Environments, 61: 483-496.

15. Loos, B. 1993. Morphological variation in Lolium (Poaceae) as a measure of species relationships. Plant Systematics and Evolution, 188: 87-99.

16. Martin, P., M. Ambrose and R. Koebner. 1994. A wheat germplasm survey uncovers salt tolerance in genotypes not exposed to salt stress in the course of their selection. Annals of Applied Biology, 39: 215-222.

17. Mohammad Alipour H. Yamchi, M.R. Bihamta, S.A. Peighambari, M. Naghavi and M. Shafiee Khorshidi. 2011. Evaluation of genetic diversity and classification of kabuli chickpea genotypes in late season drought stress. Journal of Crop Breeding, 7: 53-70 (In Persian).

18. Mohan, M., S.L. Narayanan and S. Ibrahim. 2000. Chlorophyll stability index (CSI): its impact on salt tolerance in rice. International Rice Research Notes, 25: 38-39.

19. Moreda-Pineiro, A., A. Fisher and S.J. Hill. 2003. The classification of tea according to region of origin using pattern recognition techniques and trace metal data. Journal of Food Composition and Analysis, 16: 195-211.

20. Munns, R. 2002. Comparative physiology of salt and water stress. Plant, Cell \& Environment, 25 : 239-250.

21. Munns, R. and R.A. James. 2003. Screening methods for salinity tolerance: a case study with tetraploid wheat. Plant and Soil, 253: 201-218.

22. Munns, R., R.A. James and A. Läuchli. 2006. Approaches to increasing the salt tolerance of wheat and other cereals. Journal of Experimental Botany, 57: 1025-1043.

23. Passarkli, M. 2010. Handbook of plant and crop stress. ( $3^{\text {rd }}$ edition). CRC press, 1245 pp.

24. Poustini, K. and A. Siosemardeh. 2004. Ion distribution in wheat cultivars in response to salinity stress. Field Crop Research, 85: 125-133.

25. Rabii, B. and M. Rahimi. 2009. Evaluation methods of canola genotypes grouped using the fisher linear detection function. Journal of Science and Technology of Agriculture and Natural Resourses, 47: 529-542 (In Persian).

26. Ramesh, K., B. Chandrasekaran, T. Balasubramanian, U. Bangarusamy, R. Sivasamy and N. Sankaran. 2002. Chlorophyll dynamics in rice (Oryza sativa L.) before and after flowering based on SPAD (chlorophyll) meter monitoring and its relation with grain yield. Journal of Agronomy and Crop Science, 188: 102-105.

27. Reynolds, M., A. Mujeeb-Kazi and M. Sawkins. 2005. Prospects for utilising plant-adaptive mechanisms to improve wheat and other crops in drought-and salinity-prone environments. Annals of Applied Biology, 146: 239-259.

28. Saburi, H., M. Nahvi, A. Torabi and M. Kanoni. 2008. Classification of rice varieties at different levels from the osmotic potential of sorbitol based on cluster analysis and fisher linear functions. Iranian Congress of Agronomy and Plant Breeding, 28-30 August, Karaj, Iran, Crop Science Society, 7: 327-340. 
29. Safari, P., R. Honarnejad and M. Esfahani. 2008. Assessment of genetic variation in peanuts (Arachis hypogaea L.) cultivars using Canonical Discriminant Analysis. Iranian Journal of Agriculture Research, 6: 327-334 (In Persian).

30. Salama, S., S. Trivedi, M. Busheva, A. Arafa, G. Garab and L. Erdei. 1994. Effects of NaCl salinity on growth, cation accumulation, chloroplast structure and function in wheat cultivars differing in salt tolerance. Journal of Plant Physiology, 144: 241-247.

31. Salehi, M. and M. Shekari. 2006. Factor analysis of some drought traits in lentil. In: proceeding of the Science and Crop Breeding Congress Abstracts. 286 pp (In Persian).

32. SAS Institute Inc. 2011. SAS/STAT user's guide. ${ }^{2}$ nd edition. SAS institute Inc., Cary, NC, USA, $5142 \mathrm{pp}$.

33. Schonfeld, M.A., R.C. Johnson, B.F. Carver and D.W. Mornhinweg. 1988. Water relations in winter wheat as drought resistance indicators. Crop Science, 28: 526-531.

34. Shafiee-Khorshidi, M., M.R. Bihamta, F. Khialparast and M.R. Naghavi. 2012. Assessment of genetic variation in common bean (Phaseolus vulgaris L.) genotypes under drought condition using cluster and canonical discriminant analysis (CDA). Journal of Crop Breeding, 10: 1-17 (In Persian).

35. Singh, S. and T. Singh. 2001. Correlation and path analysis in common wheat (Triticum aestivum L.) under light texture soil. Resarch on Crops, 2: 99-101.

36. SPSS, I. 2010. SPSS 19. Users Guied. Chicago, IL., USA.

37. Yeater, K. M., G.A. Bollero, D.G. Bullock, A.L. Rayburn and S. Rodriguez-Zas. 2004. Assessment of genetic variation in hairy vetch using canonical discriminant analysis. Crop Science, 44: 185-189. 


\title{
Investigation of the Genetic Diversity of Iranian bread Wheat Germplasm for Tolerance to Saline Stress
}

\author{
Amir Gholizadeh $^{1}$, Hamid Dehghani ${ }^{2}$, Ashkboos Amini ${ }^{3}$ and Omidali Akbarpour ${ }^{4}$
}

1- Ph.D Student, in Plant Breeding Department, Faculty of Agriculture, Tarbiat Modares University

2- Professor in Plant Breeding Department, Faculty of Agriculture, Tarbiat Modares University (Corresponding author: dehghanr@modares.ac.ir)

3- Assistant Professor, Seed and Plant Improvement Institute, Agricultural Research, Education and Extension Organization (AREEO), Karaj, Iran

4- Assistant Professor in Department of Agronomy and Plant Breeding, Faculty of Agriculture Lorestan University, Khorramabad, Iran

Received: April 18, $2017 \quad$ Accepted: July 1, 2017

\begin{abstract}
Salinity stress is one of the major abiotic stresses in arid and semi-arid regions of the world, such as Iran. High genetic diversity for salinity tolerance has been observed in Iranian bread wheat genotypes. In order to analyze genetic diversity and determine the most effective characteristics on salinity tolerance, 110 bread wheat genotypes were evaluated in two conditions (non-stress and saline stress) at the research field of the National Salinity Research Center (NSRC). The salinity of water used in irrigation in stress and non-stress conditions was 10 and 2 ds.m-1, respectively. The results showed that there was a significant genetic variation between studied genotypes. According to cluster analysis based on agronomical and morphological traits, genotypes were divided into 4 categories in both non-stress and stress conditions. According to the results of the means comparison of the groups in non-stress and saline stress conditions, the genotypes No. 2, 5, 7, 8, 9, 10, 11, 12, 13, 31, 35, 38, 73, 81, 97 and 98 were identified as the most salinity-tolerant genotypes. These genotypes can be utilized for salt-affected areas and also as donor parents in wheat breeding programs for further improvement of germplasm for salinity tolerance. Also, the results of factor analysis in saline stress condition indicated a positive relationship between biological yield, harvest index and chlorophyll content with seed yield. Generally, it can be concluded that chlorophyll content trait due to the low cost and easy and non-destructive measurement than other traits could be used as a suitable criterion in selecting for increased seed yield in saline stress conditions in field.
\end{abstract}

Keywords: Cluster analysis, Factor analysis, Genetic diversity, Salinity stress, Wheat 